\title{
Can Multivariate GARCH Models Really Improve Value-at-Risk Forecasts?
}

\author{
$\underline{\text { C.S. Sia }}^{\mathrm{a}}$ and F. Chan ${ }^{\mathrm{a}}$ \\ ${ }^{a}$ School of Economics and Finance, Curtin University \\ Email: chow.sia@cbs.curtin.edu.au
}

\begin{abstract}
This paper evaluates the performance of multivariate conditional volatility models in forecasting Value-at-Risk (VaR). The paper considers the Constant Conditional Correlation (CCC) model of Bollerslev (1990), and models that allow dynamic conditional correlation such as the Dynamic Conditional Correlation (DCC) model of Engle (2002) and the Time-Varying Conditional Correlation (TVC) model of Tse and Tsui (2002). While the underlying assumptions vary between these models, their common objective is to model volatility for multiple assets by capturing their possible interactions. Thus, they provide more information about the underlying assets that could not be recovered by univariate models. However, the practical usefulness of these models are limited by their complexity as the number of asset increases. The paper aims to examine this trade-off between simplicity and extra information by applying these models to forecast VaR for a portfolio of the Australian dollar with twelve other currencies. This provides some insight into the practical usefulness of the additional information for purposes of risk management.
\end{abstract}

Keywords: Value-at-Risk (VaR), Multivariate GARCH 


\section{INTRODUCTION}

Modelling volatility in financial time series has been an important research area in the past decades. The family of Autoregressive Conditional Heteroskedasticity (ARCH) model was first introduced by Engle (1982) who laid the foundation for a new approach to describe and forecast conditional variance for financial time series. Subsequently, numerous variants and extensions of ARCH models have been proposed. See for examples, the Generalized ARCH (GARCH) model of Bollerslev (1986) and its asymmetric extension by Glosten, Jagannathan, and Runkle (1993).

In many financial applications, conditional covariance and correlations play a direct and important role in volatility forecasting. A bank is very likely to trade with large and complex portfolios daily. It is unlikely that the asset returns in a portfolio would move independently of each other. Therefore, understanding their correlation structures is essential in deriving sensible investment strategies to maximize returns while minimizing risk. Most of the existing univariate volatility models focus on the dynamics of a single time series and they do not provide any information on the potential dependency between asset returns within a portfolio. It is worth noting that the correlation between asset returns may be driven by individual heterogeneity as well as any potential common factors. This implies that the correlation structures may be time-varying. For example, the correlation between Standard \& Poor's 500 (S\&P 500) and Nikkei 225 is likely to be different before and after the Global Financial Crisis (GFC). The correlation before the crisis may be driven by normal market condition whereas the GFC forms a single factor that caused significant changes in the correlation between the two indices.

To capture the conditional covariance and correlations for the different type of assets in a portfolio, many researchers expanded the univariate to multivariate volatility models. McAleer (2005) pointed out that one important aspect in modelling financial volatility is to study multivariate extensions of the conditional volatility models. Bollerslev, Engle, and Wooldridge (1988) proposed the diagonal vector ARCH (DVEC) model that is a direct extension of the univariate Generalized ARCH (GARCH) model to multivariate model. Other alternative approaches for achieving more parsimonious and empirically tractable multivariate volatility models are the Constant Conditional Correlation (CCC) model of Bollerslev (1990); Baba, Engle, Kraft and Kroner (BEKK) model described by Engle and Kroner (1995); the Dynamic Conditional Correlation (DCC) model of Engle (2002); the Time-Varying Correlation (TVC) model of Tse and Tsui (2002); the Vector ARMA-GARCH (VARMA-GARCH) model of Ling and McAleer (2003); and the VARMA-asymmetric GARCH (VARMA-AGARCH) model of McAleer, Hoti, and Chan (2009). However, the practical usefulness of these models can be affected by 'the curse of dimensionality' (see Caporin and McAleer 2014). That is, the number of parameters increases dramatically in these models as the number of asset increases.

There are a huge number of studies that estimate VaR forecasts using multivariate GARCH models. Hsu Ku and Wang (2008) examined the performance of multivariate GARCH models, namely the CCC, DCC and BEKK models, in terms of VaR violations on a portfolio of foreign exchange rates. The DCC model is considered to be the best model that offers a better forecasting performance among the other two models in estimating VaR. da Veiga, Chan, and McAleer (2011) used both CCC and DCC models on the portfolios of Chinese A and Chinese B stock returns. On one hand, DCC model provides a lower number of violations than the CCC model. On the other hand, CCC model tends to generate a lower amount of daily capital charges than the DCC model. Consequently, they showed that a more severe penalty structure is probably desirable to discourage banks from choosing forecasting models that underestimate VaR. In particular, they proposed a new penalty structure that is based on the magnitude of violations instead of the current penalty structure that is based on the number of violations. An appropriate penalty structure may encourage banks to improve their risk models in forecasting VaR more precisely. While, Bauwens and Laurent (2005) proposed a multivariate skewed-t distribution for multivariate GARCH models on the portfolios of the US stock returns and foreign exchange rates. They found that the multivariate GARCH models under multivariate skewed-t distribution improves the performance of VaR forecasts. Nevertheless, these studies showed that accommodating timevarying conditional correlations improves the forecasting performance of VaR.

This paper is outlined as follows. The structural properties of the CCC and DCC models and the market risk capital requirements by the Basel Accord are provided in Section 2. Section 3 describes the data and discusses the empirical results for the performance of VaR forecasts. Section 4 concludes the paper.

\section{CONDITIONAL VOLATILITY MODELS AND VAR FORECASTS}

Consider the following model: 
$\Phi(L) r_{t}=\Theta(L) \varepsilon_{t} \quad \varepsilon_{t}=D_{t} \eta_{t} \quad \eta_{t} \sim M V(0, I) \quad D_{t}=\operatorname{diag}\left(h_{1 t}, \ldots, h_{k t}\right)$

where $r_{t}=\left(r_{1 t}, \ldots, r_{k t}\right)$ ' is a $k \times 1$ vector of asset returns and $t=1, \ldots, T$, with $L$ denotes the lag operator such that for any time series $y_{t}, L y_{t}=y_{t-1} . \Phi(L)=I-\sum_{i=1}^{p} \phi_{i} L^{i}$ and $\Theta(L)=I+\sum_{i=1}^{q} \theta_{i} L^{i}$ are the lag polynomials of order $p$ and $q$, respectively. $\eta_{t}$ is a $k \times 1$ independently and identically distributed multivariate random vector with zero mean and identity variance-covariance matrix.

Following the model as defined in equation (1), the conditional variance and covariance matrix of $r_{t}$ is $\Omega_{t}=D_{t} \Gamma_{t} D_{t}$, where $\Gamma_{t}=\mathrm{E}_{t}\left(\eta_{t} \eta_{t}{ }^{\prime}\right)$ denotes $k \times k$ matrix of the conditional correlations between the conditional shocks. $\mathrm{E}$ and $\mathrm{E}_{t}$ denotes the unconditional and conditional expectation with respect to the information set at time $t$, respectively. The CCC model assumes that the conditional correlations are constant over time. Hence, $\Gamma=\left\{\rho_{i j}\right\}$ is a constant conditional correlation matrix with $\rho_{i j}=\rho_{j i}$. Engle (2002) and Tse and Tsui (2002) proposed the DCC model and the TVC model, respectively, to allow the conditional correlations to be time-varying, so that the conditional variance and covariance matrix of $r_{t}$ is time-varying. In that case, the dynamic of volatility depends on the specification of $\Omega_{t}$.

The parameters in these models are typically estimated by Quasi-Maximum Likelihood Estimator (QMLE), which is defined to be:

$$
\hat{\theta}=\underset{\theta \in \Lambda}{\arg \max }\left(-\frac{T}{2} \sum_{t=1}^{T} \log \left|\Omega_{t}\right|+\varepsilon_{t}^{\prime} \Omega_{t}^{-1} \varepsilon_{t}\right)
$$

Where $\theta$ denotes the parameters to be estimated in the conditional log-likelihood function. $\left|\Omega_{t}\right|$ denotes the determinant of $\Omega_{t}$. See McAleer (2005) and McAleer et al. (2008) for more technical discussions on this class of models, including the sufficient conditions for the existence of moments and the sufficient conditions for consistency and asymptotic normality of QMLE.

Following equation (1), the $\mathrm{VaR}$ forecast at $\alpha=0.01$ for asset $i$ at time $t+1$ can be obtained as:

$$
\operatorname{VaR}_{i, t+1}^{m}=\mathrm{E}_{t}\left(r_{i, t+1}\right)+q_{\alpha, d} \sqrt{h_{i, t+1}^{m}}
$$

where $\mathrm{E}_{t}\left(r_{i, t+1}\right)$ is the forecast of the asset $i$ 's return based on the information at time $t, q_{\alpha, d}$ is the critical value based on the significant level of VaR and the distribution of $\eta_{t}$. Although $\eta_{t}$ is typically assumed to be normally distributed, a student-t distribution with $\delta$ degrees of freedom can be used an alternative. $\sqrt{h_{i, t+1}^{m}}$ is the estimated standard deviation of $\mathrm{E}_{t}\left(r_{i, t+1}\right)$ with $m$ denotes the model used. Noted that the superscripts "std" and "norm" denotes estimates assuming a normal distributed return and a t-distributed return.

The current regulatory framework requires banks that use their own internal risk models to calculate the VaR on a daily basis at 99 percent confidence level. Backtesting procedures have been used to evaluate the performance of VaR models. As such, the market risk capital requirements are determined as follows (Basel Committee on Banking Supervision 2011):

1. A bank must backtest its internal VaR models over the previous 250 trading days.

2. To monitor the frequency of violations, the number of times that the actual losses exceed VaR forecasts are calculated. Subsequently, the percentage of violations can also be calculated. A good model will have a percentage of violation that is very close to one percent and should lead to correct estimation of market risk at every point in time. A VaR model that overestimates market risk will lead to insufficient violations and requires a large amount of capital. On the other hand, a VaR model that underestimates market risk will be penalized by the regulator due to excessive violations. 
3. The market risk capital charge (MRCC) is set either at the lower VaR of the previous day or the average $\mathrm{VaR}$ of the previous 60 days trading days, multiplied by a scaling factor of $(3+\mathrm{k})$. The scaling factor calculates the probability that a violation occurs for a given day over the previous 250 trading days. It can be written as:

$$
M R C C_{t}=\min \left(\operatorname{VaR}_{\alpha, t},\left(3+k_{t}\right) \times \frac{1}{60} \sum_{i=1}^{60} \operatorname{VaR}_{\alpha, t}\right)
$$

where,

$$
k_{t}=\left\{\begin{array}{cc}
0 & V_{t}^{250} \leq 4(\text { Green }) \\
0.40-0.85 & V_{t}^{250}=5-9(\text { Yellow }) \\
1 & V_{t}^{250} \geq 10(\text { Red })
\end{array}\right.
$$

Some statistical tests are also carried out to validate VaR forecasts. These tests include Kupiec (1995) Test Until the First Failure (TUFF), followed by Christoffersen (1998) and Christoffersen, Hahn, and Inoue (2001) Unconditional Coverage (UC), Serial Independence (IND) and Conditional Coverage (CC) tests. See da Veiga, Chan, and McAleer (2011) for further discussion on these stastical tests.

\section{RESULTS}

A dataset of daily exchange rates on Australian dollar (AUD) with twelve other currencies is used. The exchange rates are US Dollar (USD), Japanese Yen (JPY), Pound Sterling (GBP), New Zealand Dollar (NZD), Korean Won (KRW), Singapore Dollar (SGD), Swiss Franc (CHF), Chinese Renminbi (CNY), Hong Kong Dollar (HKD), Indian Rupee (IDR), Malaysian Ringgit (MYR), and New Taiwan Dollar (TWD). These exchange rates are collected from Thomson Reuters DataStream Professional, for the period of 2 January 1984 to 31 December 2013. Using the data above, an equally-weighted portfolio of twelve assets is constructed. The sample size used for estimation is from 2 January 1984 to 31 December 2002 with 4,950 observations and the forecasting period is from 2 January 2003 to 31 December 2013 with 2,871 observations. The means of the portfolio returns for both estimation and forecast periods are close to zero. The skewness of the portfolio returns for both estimation and forecast periods are negative. While, the portfolio returns display high kurtosis and fattailed.

There are four sets of VaR forecasts estimated from the CCC-GARCH(1,1), CCC-GJR(1,1), DCC$\operatorname{GARCH}(1,1)$ and DCC-GJR $(1,1)$ models for normal distribution. The degrees of freedom set by t-density are estimated from the standardized residuals that follow $\operatorname{GARCH}(1,1)$ and $\operatorname{GJR}(1,1)$ processes utilized under normal and student-t distributions. This gives eight critical values that leads to eight sets of VaR forecasts. A total 12 sets of $\mathrm{VaR}$ forecasts are presented for comparison purposes. All VaR forecasts are constructed at $1 \%$ level. The parameter estimates in the CCC-GARCH(1,1), CCC-GJR(1,1), DCC-GARCH(1,1) and DCCGJR $(1,1)$ models are statistically significant for all currencies. As the second moment condition is satisfied, the log-moment condition is necessarily satisfied, so the QMLE is consistent and asymptotically normal.

\begin{tabular}{|c|c|c|c|c|c|}
\hline Model & Mean & Median & Minimum & Maximum & $\begin{array}{c}\text { Standard } \\
\text { Deviation }\end{array}$ \\
\hline$V a R_{n o r m}^{C C C G A R C H-N}$ & $-1.2943^{(1)}$ & -1.1560 & -5.6650 & -0.7386 & 0.5336 \\
\hline$\nabla a R_{\text {norm }}^{\text {CCCGJR-N }}$ & $-1.2956^{(1)}$ & -1.1520 & -5.6090 & -0.7479 & 0.5386 \\
\hline$\nabla a R_{s t d}^{C C C G A R C H-N}$ & $-1.5071^{(2)}$ & -1.3460 & -6.5960 & -0.8601 & 0.6214 \\
\hline$\nabla a R_{s t d}^{C C C G J R-N}$ & $-1.5072^{(2)}$ & -1.3400 & -6.5250 & -0.8700 & 0.6265 \\
\hline $\bar{V} a R_{\text {std }}^{\text {CCCGARCH }-t}$ & $-1.5199^{(3)}$ & -1.3560 & -6.5840 & -0.8696 & 0.6287 \\
\hline$\nabla a R_{s t d}^{C C C G J R-t}$ & $-1.5179^{(3)}$ & -1.3500 & -6.4680 & -0.8738 & 0.6326 \\
\hline$\forall a R_{\text {norm }}^{D C C G A R C H-N}$ & $-1.3336^{(1)}$ & -1.1860 & -6.3490 & -0.7172 & 0.5896 \\
\hline$V a R_{\text {norm }}^{D C C G J R-N}$ & $-1.3353^{(1)}$ & -1.1800 & -6.2380 & -0.7264 & 0.5952 \\
\hline$\forall a R_{s t d}^{D C C G A R C H-N}$ & $-1.5148^{(2)}$ & -1.3470 & -7.2110 & -0.8146 & 0.6697 \\
\hline$\nabla a R_{\text {std }}^{D C C G J R-N}$ & $-1.5166^{(2)}$ & -1.3400 & -7.0850 & -0.8250 & 0.6761 \\
\hline$V a R_{s t d}^{D C C G A R C H-t}$ & $-1.6029^{(3)}$ & -1.4300 & -7.4590 & -0.7922 & 0.7249 \\
\hline$\forall a R_{s t d}^{D C C G J R-t}$ & $-1.6001^{(3)}$ & -1.4200 & -7.2630 & -0.8075 & 0.7288 \\
\hline
\end{tabular}

Table 1. VaR Forecasts at $1 \%$ level 


\begin{abstract}
${ }^{(2)} \mathrm{VaR}$ forecasts are estimated from equation (6) based on a normal distribution at the degrees of freedom set by t-density
(3) VaR forecasts are estimated from equation (6) based on a student-t distribution at the degrees of freedom set by t-density

Table 1 summarizes the results for the 12 sets of VaR forecasts estimated by the CCC and DCC models. The means of VaR forecasts for the CCC and DCC models that utilized under student-t distribution appear to be lower than the means of VaR forecasts for the CCC and DCC models under the normal distribution. Hence, the student-t distribution provides more conservative VaR forecasts than a normal distribution. It can also be seen that the means of VaR forecasts estimated by the DCC models are mostly lower than the means of VaR forecasts estimated by the CCC models. In particular, $V_{a} R_{s t d}^{D C G A R C H-t}$ shows the lowest mean of VaR forecasts at -1.6029 while $\forall a R_{\text {norm }}^{\text {CCCGRCH }-N}$ shows the highest mean of VaR forecasts at -1.2943 . Hence, VaR forecasts estimated by the DCC models are crucial to improve the performance of VaR forecasts. These results also justify the use of model such as DCC to capture the time-varying conditional correlation structures in portfolio returns.
\end{abstract}

Table 2. Number and Percentage of Violations for VaR Forecasts at 1\% Level

\begin{tabular}{|c|c|c|}
\hline Model & No. of Violation & $\%$ of Violation \\
\hline$\nabla a R_{\text {norm }}^{C C C G A R H-N}$ & 71 & $2.47 \%$ \\
\hline$\nabla a R_{n o r m}^{C C C G R-N}$ & 69 & $2.40 \%$ \\
\hline$V a R_{s t d}^{C C C G A R C H-N}$ & 41 & $1.43 \%$ \\
\hline$V a R_{\text {std }}^{C C C J R-N}$ & 39 & $1.36 \%$ \\
\hline$\nabla a R_{s t d}^{C C C G A R C H-t}$ & 41 & $1.43 \%$ \\
\hline$V a R_{s t d}^{C C C G J R-t}$ & 40 & $1.39 \%$ \\
\hline 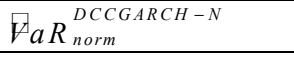 & 68 & $2.37 \%$ \\
\hline$\forall a R_{\text {norm }}^{D C C J R-N}$ & 64 & $2.23 \%$ \\
\hline$\forall a R_{s t d}^{D C C G A R C H-N}$ & 41 & $1.43 \%$ \\
\hline$V a R_{s t d}^{D C C G J R-N}$ & 39 & $1.36 \%$ \\
\hline$\overline{V a R_{s t d}^{D C C G A R C H-t}}$ & 35 & $1.22 \%$ \\
\hline$\forall a R_{s t d}^{D C C J R-t}$ & 33 & $1.15 \%$ \\
\hline
\end{tabular}

Table 2 reports the number and percentage of violations for VaR forecasts. Ideally, a good model would have a percentage of violation that is very close to one percent. A model that underestimates market risk gives a percentage of violation that is more than one percent. A model that overestimates market risk gives a percentage of violation that is less than one percent. High percentages of violations for $\forall a R_{\text {norm }}^{C C C G A R C H-N}$ and $V a R_{\text {norm }}^{C C C G J R-N}$ are observed at $2.47 \%$ and $2.40 \%$, respectively. Similarly, $\operatorname{VaR} R_{\text {norm }}^{D C C G A C H-N}$ and $\operatorname{VaR} R_{\text {norm }}^{D C C G N-N}$ present high percentages of violations at $2.37 \%$ and $2.23 \%$, respectively. A good model is given by $\nabla a R_{s t d}^{D C C G J-t}$ with a percentage of violations at 1.15 . The highest percentage of violations is given by $\nabla a R_{\text {norm }}^{C C C G A R H-N}$ at $2.47 \%$. It is worth noting that the DCC models are preferred to the CCC models given that the DCC models provide the percentages of VaR violations that are closer to one percent. Also, the VaR violations under student- $t$ distribution always give fewer violations than VaR violations under the normal distribution.

Table 3. Backtesting Results for VaR Forecasts at $1 \%$ level

\begin{tabular}{|c|c|c|c|c|}
\hline Model & TUFF $^{(1)}$ & $\mathrm{UC}^{(1)}$ & Ind $^{(2)}$ & $\mathrm{CC}^{(2)}$ \\
\hline $\bar{V} a R_{\text {norm }}^{\text {CCCGACH }-N}$ & 0.3715 & 44.6241 & 12.9469 & $\mathbf{5 7 . 5 7 1 0}$ \\
\hline$V a R_{\text {norm }}^{C C C G J-N}$ & 0.3715 & 41.0007 & 13.7388 & 54.7394 \\
\hline$\forall a R_{s t d}^{C C C G A R C H-N}$ & 0.3715 & 4.6920 & 5.2691 & 9.9611 \\
\hline$V a R_{s t d}^{C C C G J R-N}$ & 0.3715 & 3.3500 & 2.4869 & 5.8368 \\
\hline$\nabla a R_{s t d}^{C C C G A R C H-t}$ & 0.3715 & 4.6920 & 5.2691 & 9.9611 \\
\hline$\forall a R_{s t d}^{C C C G J R-t}$ & 0.3715 & 3.9956 & 2.3324 & 6.3280 \\
\hline$\forall a R_{\text {norm }}^{D C C G A C H-N}$ & 0.3715 & 39.2333 & 10.6979 & 49.9312 \\
\hline $\begin{array}{l}a R_{\text {norm }}^{D C C G R-N} \\
\end{array}$ & 0.3715 & 32.4696 & 8.7800 & 41.2496 \\
\hline$\nabla a R_{\text {std }}^{D C C G A R C H-N}$ & 0.3715 & 4.6920 & 2.1846 & 6.8766 \\
\hline$V a R_{s t d}^{D C C G J R-N}$ & 0.3715 & 3.3500 & 2.4869 & 5.8368 \\
\hline
\end{tabular}




\begin{tabular}{|c|c|c|c|c|}
\hline$V a R_{s t d}^{D C C G A R C H-t}$ & 0.3715 & 1.3011 & 3.1780 & 4.4791 \\
\hline$\nabla a R_{s t d}^{D C C G J R-t}$ & 0.3715 & 0.6178 & 3.5724 & 4.1901 \\
\hline
\end{tabular}

The results from TUFF, UC, Ind and CC tests are given in Table 3. The TUFF results for all models lead to correct acceptance of the test at a constant value of 0.3715 . It can be seen that both CCC and DCC models utilized under a normal distribution, fail UC, Ind and CC tests. This suggests that the VaR violations performed by these models are serially dependent. On the other hand, $\forall a R_{s t d}^{C C C G J R-N}, \forall a R_{s t d}^{D C C G J R-N}, \forall a R_{s t d}^{D C C G A R C H-t}$, and $\forall a R_{s t d}^{D C C G J R-t}$ pass UC, Ind and CC tests. This shows that the VaR violations are likely to be independent and that a VaR violation today should not provide any information about whether or not a VaR violation will occur tomorrow. While, $\forall a R_{\text {norm }}^{C C C G A R C H-N}$, , $\nabla a R_{\text {std }}^{C C C G A R C H-t}, \forall a R_{s t d}^{C C C G J R-t}$, and $\forall a R_{s t d}^{D C C G A R C H-N}$, fail the UC and $\mathrm{CC}$ tests but pass Ind test.

Table 4. Market Risk Capital Charges

\begin{tabular}{|c|c|c|c|c|c|}
\hline Model & Mean & Median & Minimum & Maximum & $\begin{array}{c}\text { Standard } \\
\text { Deviation }\end{array}$ \\
\hline$V a R_{n o r m}^{C C C G A R C H-N}$ & -4.79 & -4.06 & -22.66 & -2.44 & 2.5215 \\
\hline$V a R_{n o r m}^{C C C G J R-N}$ & -4.77 & -4.01 & -22.43 & -2.46 & 2.5406 \\
\hline$V a R_{s t d}^{C C C G A R C H-N}$ & -5.18 & -4.38 & -24.74 & -2.84 & 2.6551 \\
\hline$V a R_{s t d}^{C C C G J R-N}$ & -5.12 & -4.36 & -23.82 & -2.86 & 2.5406 \\
\hline$V a R_{s t d}^{C C C G A R C H-t}$ & -5.22 & -4.41 & -24.61 & -2.84 & 2.6874 \\
\hline$V a R_{s t d}^{C C C G J R-t}$ & -5.19 & -4.38 & -24.26 & -2.88 & 2.6821 \\
\hline$\forall a R_{\text {norm }}^{\text {DCCGARCH }-N}$ & -4.87 & -4.05 & -25.39 & -2.39 & 2.7387 \\
\hline VaR ${ }_{\text {norm }}^{D C C G J R-N}$ & -4.81 & -4.01 & -24.02 & -2.40 & 2.6061 \\
\hline$V a R_{s t d}^{D C C G A R C H-N}$ & -5.19 & -4.45 & -26.32 & -2.71 & 2.6897 \\
\hline$V a R_{\text {std }}^{D C C G J R-N}$ & -5.15 & -4.34 & -25.86 & -2.73 & 2.7088 \\
\hline$\forall a R_{s t d}^{D C C G A R C H-t}$ & -5.41 & -4.63 & -26.10 & -2.78 & 2.7875 \\
\hline$V a R_{s t d}^{D C C G J R-t}$ & -5.31 & -4.59 & -24.69 & -2.81 & 2.5191 \\
\hline
\end{tabular}

Table 4 shows the market risk capital charges from equations (4) and (12). Berkowitz and O'Brien (2002) and Pérignon, Deng, and Wang (2008) showed that banks tend to report high VaR forecasts that lead to an excessive amount of capital charges. In any case, there is an opportunity cost of misestimating VaR. Hence, pursuing a correct VaR model that can lead to the precision of determining minimum capital charges is crucial for banks and the regulator. In this case, $V_{a} R_{\text {std }}^{D C C G A R C H-t}$ provides the lowest mean of market risk capital charges at -5.41. While, the lowest capital charge is given by $\forall a R_{s t d}^{D C C G A R C H-N}$ at -26.32 . This is mostly expected due to the extreme negative returns during the GFC of 2008 where higher amount of capital charges are imposed to protect banks to cover from the worst possible trading losses. On the other hand, the highest capital charge is presented by $\nabla a R_{n o r m}^{D C C G A R C H-N}$ at -2.39 , followed by $\operatorname{Va} R_{n o r m}^{D C C G J R-N}$ at -2.40 . This generally occur during periods of low volatility in the foreign exchange market.

\section{CONCLUSION}

This paper emphasizes the importance of accommodating time-varying conditional correlations in forecasting $\mathrm{VaR}$. These findings are crucial for banks and the regulator since a correct VaR model leads to increase efficiency in measuring market risk, hence leading to determine minimum capital requirements. In this paper, two multivariate volatility models, namely CCC and DCC models, are considered to forecast VaR. The results show that a student-t distribution gives more robust estimation of VaR forecasts than a normal distribution, given that the foreign exchange returns exhibit heavy tails. The results also find that the DCC models provide more conservative VaR forecasts than CCC models with the DCC models have lower numbers and percentages of $\mathrm{VaR}$ violations. Consequently, it is reasonable to suggest that time-varying conditional correlations cannot be ignored in forecasting VaR. Also, CCC models deliver a higher amount of capital charges compared to the DCC models. These results are consistent with the empirical findings by da Veiga, Chan, and McAleer (2011). 
Incorporating multivariate volatility in VaR models is not straightforward where there are many other factors to be considered. These models raise some difficulties in practice, where banks trade with relatively large and complex portfolios that are unlikely to change daily. This implies that each day, the banks would have to compute a series of historical data for the new portfolios to estimate VaR. This may create additional costs to the banks. Instead of using these models, banks appear to be taking less computationally demanding alternatives. Banks prefer to use a simple VaR model that aggregates all of the risks of a portfolio into a single number, which is suitable for use in the boardroom, reporting to the regulator and disclosure in their financial reports. Nonetheless, multivariate volatility models play a significant role in the study of VaR as they are very useful to measure and manage market risk.

\section{REFERENCES}

Basel Committee on Banking Supervision. (2011). Basel III: A global regulatory framework for more resilient banks and banking systems - revised version. Basel, Switzerland: Bank for International Settlements.

Bauwens, L., and S. Laurent. (2005). A new class of multivariate skew densities, with application to generalized autoregressive conditional heteroscedasticity models. Journal of Business \& Economic Statistics, 23(3), 346-354.

Berkowitz, J., and J. O'Brien. (2002). How accurate are value-at-risk models at commercial banks? Journal of Finance, 57(3), 1093-1111.

Bollerslev, T. (1986). Generalized autoregressive conditional heteroscedasticity. Journal of Econometrics, 31, 307-327.

Bollerslev, T. (1990). Modelling the coherence in short-run nominal exchange rate: a multivariate generalized arch approach. Review of Economics \& Statistics, 72(3), 498-505.

Bollerslev, T., R. F. Engle, and J. M. Wooldridge. (1988). A capital asset pricing model with time-varying covariances. Journal of Political Economy, 96(1), 116-131.

Caporin, M., and M. McAleer. (2014). Robust ranking of multivariate GARCH models by problem dimension. Computational Statistics \& Data Analysis, 76, 172-185.

Christoffersen, P. (1998). Evaluating interval forecasts. International Economic Review, 39, 841-862.

Christoffersen, P., J. Hahn, and A. Inoue. (2001). Testing and comparing value-at-risk measures. Journal of Empirical Finance, 8(3), 325-342.

da Veiga, B., F. Chan, and M. McAleer. (2011). It pays to violate: how effective are the Basel Accord penalties in encouraging risk management? Accounting \& Finance, 52(1), 95-116.

Engle, R. F. (1982). Autoregressive conditional heteroscedasticity with estimates of the variance of United Kingdom inflation. Econometrica, 50, 987-1007.

Engle, R. F. (2002). Dynamic conditional correlation: a simple class of multivariate generalized autoregressive conditional heteroskedasticity models. Journal of Business \& Economic Statistics, 20(3), 339-350.

Engle, R. F., and K. F. Kroner. (1995). Multivariate simultaneous generalized ARCH. Econometric Theory, 11(1), 122-150.

Glosten, L. R., R. Jagannathan, and D. E. Runkle. (1993). On the relation between the expected value and the volatility of the nominal excess return on stocks. Journal of Finance, 48(5), 1779-1801.

Hsu Ku, Y. H., and J. J. Wang. (2008). Estimating portfolio value-at-risk via dynamic conditional correlation MGARCH model - an empirical study on foreign exchange rates. Applied Economics Letters, 15(7), 533-538.

Kupiec, P. (1995). Techniques for verifying the accuracy of risk measurement models. Journal of Derivatives, 3, 73-84.

Ling, S., and M. McAleer. (2003). Asymptotic theory for a vector ARMA-GARCH model. Econometric Theory, 19(2), 280-310.

McAleer, M. (2005). Automated inference and learning in modeling financial volatility. Econometric Theory, 21(01), 232-261.

McAleer, M., F. Chan, S. Hoti, and O. Lieberman. (2008). Generalized autoregressive conditional correlation. Econometric Theory, 24(06), 1554-1583.

McAleer, M., S. Hoti, and F. Chan. (2009). Structure and asymptotic theory for multivariate asymmetric conditional volatility. Econometric Reviews, 28(5), 422-440.

Pérignon, C., Z. Y. Deng, and Z. J. Wang. (2008). Do banks overstate their value-at-risk? Journal of Banking \& Finance, 32(5), 783-794.

Tse, Y. K., and A. K. C. Tsui. (2002). A multivariate generalized autoregressive conditional heteroscedasticity model with time-varying correlations. Journal of Business \& Economic Statistics, 20(3), 351-362. 